\title{
On sums of coefficients of polynomials related to the Borwein conjectures
}

\author{
Ankush Goswami ${ }^{1}$ - Venkata Raghu Tej Pantangi ${ }^{2}$
}

Received: 29 April 2020 / Accepted: 22 October 2020 / Published online: 18 February 2021

(C) The Author(s) 2021

\begin{abstract}
Recently, Li (Int J Number Theory, 2020) obtained an asymptotic formula for a certain partial sum involving coefficients for the polynomial in the First Borwein conjecture. As a consequence, he showed the positivity of this sum. His result was based on a sieving principle discovered by himself and Wan (Sci China Math, 2010). In fact, Li points out in his paper that his method can be generalized to prove an asymptotic formula for a general partial sum involving coefficients for any prime $p>3$. In this work, we extend Li's method to obtain asymptotic formula for several partial sums of coefficients of a very general polynomial. We find that in the special cases $p=3,5$, the signs of these sums are consistent with the three famous Borwein conjectures. Similar sums have been studied earlier by Zaharescu (Ramanujan J, 2006) using a completely different method. We also improve on the error terms in the asymptotic formula for Li and Zaharescu. Using a recent result of Borwein (JNT 1993), we also obtain an asymptotic estimate for the maximum of the absolute value of these coefficients for primes $p=2,3,5,7,11,13$ and for $p>15$, we obtain a lower bound on the maximum absolute value of these coefficients for sufficiently large $n$.
\end{abstract}

Keywords Borwein conjectures $\cdot$ Positivity $\cdot$ Multinomial $\cdot$ Sieve

Mathematics Subject Classification 11P81 $11 \mathrm{P} 83 \cdot 11 \mathrm{P} 84$

The research of the first author was supported by grant SFB F50-06 of the Austrian Science Fund (FWF).

Ankush Goswami

ankushgoswami3@gmail.com; ankush.goswami@risc.jku.at

Venkata Raghu Tej Pantangi

pvrt1990@gmail.com; pantangi@sustech.edu.cn

1 Research Institute for Symbolic Computation (RISC), JKU, Linz, Austria

2 Department of Mathematics, Southern University of Science and Technology (SUSTECH),

Shenzhen, China 


\section{Introduction}

In 1990, Peter Borwein (see [1]) empirically discovered quite a number of mysteries involving sign patterns of coefficients of certain polynomials. The most easily stated are the following:

Conjecture 1.1 (First Borwein conjecture) For the polynomials $A_{n}(q), B_{n}(q)$ and $C_{n}(q)$ defined by

$$
\prod_{j=1}^{n}\left(1-q^{3 j-2}\right)\left(1-q^{3 j-1}\right)=A_{n}\left(q^{3}\right)-q B_{n}\left(q^{3}\right)-q^{2} C_{n}\left(q^{3}\right)
$$

each has non-negative coefficients.

Conjecture 1.2 (Second Borwein conjecture) For the polynomials $\alpha_{n}(q), \beta_{n}(q)$ and $\gamma_{n}(q)$ defined by

$$
\prod_{j=1}^{n}\left(1-q^{3 j-2}\right)^{2}\left(1-q^{3 j-1}\right)^{2}=\alpha_{n}\left(q^{3}\right)-q \beta_{n}\left(q^{3}\right)-q^{2} \gamma_{n}\left(q^{3}\right)
$$

each has non-negative coefficients.

Conjecture 1.3 (Third Borwein conjecture) For the polynomials $v_{n}(q), \phi_{n}(q), \chi_{n}(q)$, $\psi_{n}(q)$ and $\omega_{n}(q)$ defined by

$$
\begin{aligned}
& \prod_{j=1}^{n}\left(1-q^{5 j-4}\right)\left(1-q^{5 j-3}\right)\left(1-q^{5 j-2}\right)\left(1-q^{5 j-1}\right) \\
& \quad=v_{n}\left(q^{5}\right)-q \phi_{n}\left(q^{5}\right)-q^{2} \chi_{n}\left(q^{5}\right)-q^{3} \psi\left(q^{5}\right)-q^{4} \omega_{n}\left(q^{5}\right)
\end{aligned}
$$

each has non-negative coefficients.

Recently, Wang [6] gave an analytic proof of the First Borwein conjecture using saddle point method. His proof, besides other things, relied on a formula of Andrews [1, Theorem 4.1] and the following recursive relations [1, Theorem 3.1]:

$$
\begin{aligned}
& A_{n}(q)=\left(1+q^{2 n-1}\right) A_{n-1}(q)+q^{n} B_{n-1}(q)+q^{n} C_{n-1}(q), \\
& B_{n}(q)=q^{n-1} A_{n-1}(q)+\left(1+q^{2 n-1}\right) B_{n-1}(q)-q^{n} C_{n-1}(q), \\
& C_{n}(q)=q^{n-1} A_{n-1}(q)+q^{n-1} B_{n-1}(q)-\left(1+q^{2 n-1}\right) C_{n-1}(q) .
\end{aligned}
$$

Let $p \geq 3$ be a prime and $s, n \in \mathbb{N}$. Consider the polynomial

$$
T_{p, s, n}(q):=\prod_{j=0}^{n} \prod_{k=1}^{p-1}\left(1-q^{p j+k}\right)^{s}
$$


For $s=1$, Borwein [2] obtained an asymptotic estimate for $\left\|T_{p, 1, n}(q)\right\|_{|q|=1}=$ $\sup _{|q|=1}\left|T_{p, 1, n}(q)\right|$ when $p=2,3,5,7,11$ and 13 . However for $p>15$, he obtained an asymptotic lower bound for this quantity.

It is clear that $d_{p, s, n}:=\operatorname{deg} T_{p, s, n}=p(p-1) s(n+1)^{2} / 2$. Define the coefficients $t_{i, p, s}$ by

$$
\begin{aligned}
T_{p, s, n}(q) & :=\sum_{i=0}^{d_{p, s, n}} t_{i, p, s} q^{i} \\
& =T_{0, p, s, n}\left(q^{p}\right)+q T_{1, p, s, n}\left(q^{p}\right)+\cdots+q^{p-1} T_{p-1, p, s, n}\left(q^{p}\right)
\end{aligned}
$$

where $T_{i, p, s, n}(q) \in \mathbb{Z}[q]$. Given a polynomial $f(x)$, by $\left[x^{j}\right] f(x)$, we denote the coefficient of $x^{j}$ in $f(x)$. Let $a, d \in \mathbb{Z}$. In what follows, assume $p \mid a$ and let $S_{a, d, j, p}$ denote the arithmetic progression

$$
S_{a, d, j, p}:=\{a m+d: m \in \mathbb{Z}\}, \text { with } d \equiv j \quad(\bmod p) .
$$

Put $a=p \ell$ and consider the following finite sum of coefficients over $S_{a, d, j, p}$ :

$$
\sum_{\substack{i \geq 0 \\ i \in S_{p \ell, d, j, p}}} t_{i, p, s}=\sum_{\substack{i \geq 0 \\ i \in S_{p \ell, d, j, p}}}\left[q^{i-j}\right] T_{j, p, s, n}\left(q^{p}\right) .
$$

In [7, p. 98, Theorem 1], Zaharescu obtained an asymptotic formula for the sum in (1.4) when $\ell$ is an odd prime $\leq n+1$ and $\ell \neq p$. As a result, when $\ell \leq c(n+1)$ with $0<c<1$, he showed positivity (resp. negativity) of the sum in (1.4) when $j=0$ (resp. $j \neq 0$ ) for large $n$.

As Zaharescu points out in his paper, it is interesting to obtain positivity (or negativity) of the above sum for larger values of $\ell$. When $\ell \gg(n+1)^{2}$ (with implied constant larger than 1), one can isolate each individual terms in the sum (1.4). We note here that the main disadvantage of his asymptotic formula is the error term, which is large. This forces him to choose a $\ell \ll n+1$ which ensures that the main term is bigger than the error term, thereby showing positivity or negativity of the sums.

For $(p, s, \ell, j)=(3,1, n+1,0), \mathrm{Li}$ [4] obtained an asymptotic formula for the sum in (1.4) using a new sieve technique discovered by himself and Wan [3]. If we denote by $t_{i, 3,1}=a_{i}$, then Li proved that

Theorem 1.1 (Li) For $0 \leq j \leq(n+1)$ we have

$$
\sum_{\ell^{\prime}=0}^{n} a_{3 j+3 \ell^{\prime}(n+1)}=\frac{2 \cdot 3^{n}}{n+1}(1+o(1)) .
$$

In particular, we have

$$
\sum_{\ell^{\prime}=0}^{n} a_{3 j+3 \ell(n+1)}>0 .
$$


Indeed, the error term in Li's asymptotic formula [4, p. 4, Theorem 1.5] is much better than Zaharescu's which enabled him to prove the positivity of the sum in Theorem 1.1.

The purpose of this paper is to extend Li's results by obtaining asymptotic formula for the sums in (1.4) in the case $\ell=n+1$ for all $p, s, j$. As a consequence, we obtain positivity (or negativity) of the sums in (1.4) for large $n$. Thus, for $p=3,5$, we obtain asymptotic formula for the partial sums of coefficients involving polynomials in Conjectures 1.1-1.3. This in turn shows that the sums are positive (or negative) for all $n>0$ (see Corollaries 3.5.2-3.5.4). We also improve on the error terms in Li's and Zaharescu's asymptotic formula. Using a recent result of Borwein [2], we also obtain an asymptotic estimate for the maximum absolute coefficients of $T_{p, s, n}(q)$ only in the case $p=2,3,5,7,11,13$; however for $p>15$ we obtain an asymptotic lower bound for the maximum absolute coefficients.

This paper is organized as follows. In Sect. 2 we introduce a few notations, conventions and do some basic counting. In Sect. 3 we state our main results. In Sect. 4 we recall Li and Wan's [3] sieving principle and also establish a few basic results. Finally in Sect. 5 we obtain the proofs of our main results.

\section{Notation, conventions and basic counting}

Let $n \in \mathbb{N}$ and $p \geq 3$ be a prime. Set $N_{p}=(n+1) p$ and $D_{p}=\{1,2, \ldots, p-1, p+$ $1, \ldots, 2 p-1, \ldots, p n+1, \ldots, p n+p-1\}$. We define the following:

$$
\begin{aligned}
& \mathscr{C}_{e, p, s}(j, n):=\#\left\{\searrow_{i=1}^{s} V_{i} \subset D_{p}^{s}: \sum_{i=1}^{s}\left|V_{i}\right| \equiv 0(\bmod 2), \sum_{i=1}^{s} \sum_{x_{v_{i}} \in V_{i}} x_{v_{i}}=j\right\}, \\
& \mathscr{C}_{o, p, s}(j, n):=\#\left\{\searrow_{i=1}^{s} V_{i} \subset D_{p}^{s}: \sum_{i=1}^{s}\left|V_{i}\right| \equiv 1(\bmod 2), \sum_{i=1}^{s} \sum_{x_{v_{i}} \in V_{i}} x_{v_{i}}=j\right\} .
\end{aligned}
$$

It is now apparent that

$$
t_{j, p, s}=\mathscr{C}_{e, p, s}(j, n)-\mathscr{C}_{o, p, s}(j, n)
$$

We note that in the case $s=1, \mathscr{C}_{e, p, 1}(j, n)$ (respectively $\mathscr{C}_{o, p, 1}(j, n)$ ) counts the number of partitions of $j$ into an even (respectively odd) number of distinct nonmultiples of $p$.

As in [4], we shift the problem to that of counting the size of certain subsets of the group $G=\mathbb{Z}_{N_{p}}$. We note that $G \backslash D_{p}$ is a subgroup of index $p$. Given $0 \leq$ $k_{1}, k_{2}, \ldots, k_{s} \leq\left|D_{p}\right|$ and $0 \leq b<N_{p}$, define

$$
\begin{aligned}
& M_{p, s, n}\left(k_{1}, k_{2}, \ldots, k_{s} ; b\right) \\
& :=\#\left\{\searrow_{i=1}^{s} V_{i} \subset D_{p}^{s}:\left|V_{1}\right|=k_{1}, \ldots,\left|V_{s}\right|=k_{s}, \sum_{i=1}^{s} \sum_{x_{v_{i}} \in V_{i}} x_{v_{i}} \equiv b\left(\bmod N_{p}\right)\right\},
\end{aligned}
$$


and set

$$
M_{p, s, n}(b)=\sum_{0 \leq k_{1}, k_{2}, \ldots, k_{s} \leq\left|D_{p}\right|}(-1)^{k_{1}+k_{2}+\cdots+k_{s}} M_{p, s, n}\left(k_{1}, k_{2}, \ldots, k_{s} ; b\right) .
$$

From (1.4) and (2.1), we see that if $b \equiv j(\bmod p)$ then the following are equivalent:

$$
\begin{aligned}
M_{p, s, n}(b) & =\sum_{\substack{0 \leq i \leq d_{p, s, n} \\
i \in S_{N_{p}, b, j, p}}} t_{i, p, s}=\sum_{0 \leq \ell \leq B_{p, s}(b)} t_{N_{p} \ell+b, p, s} \\
& =\sum_{0 \leq \ell \leq B_{p, s}(b)}\left[q^{b-j+\ell N_{p}}\right] T_{j, p, s, n}\left(q^{p}\right),
\end{aligned}
$$

where $B_{p, s}(b):=\left\lfloor\left(d_{p, s, n}-b\right) / N_{p}\right\rfloor$. For ease of notation, we will mostly use the second sum in (2.2) for $M_{p, s, n}(b)$.

We next introduce a few more notations. Let $(x)_{k}:=x(x-1)(x-2) \cdots(x-k+1)$ denote the falling factorial. Let $\hat{G}$ be the set of complex-valued linear characters of $G$. By $\psi_{0}$, we denote the trivial character in $\hat{G}$. Let $X_{p, k}=D_{p}^{k}$ and $\bar{X}_{p, k}$ denote the subset of all tuples in $D_{p}^{k}$ with distinct coordinates.

\section{Main results}

Our main results are below.

Theorem 3.1 With $M_{p, s, n}(b)$ defined as in (2.2) and $b \in \mathbb{Z}_{N_{p}}$ we have

$$
\left|M_{p, s, n}(b)-\frac{\Sigma_{p, s, n, b}}{n+1}\right| \leq p^{s(n+1) / 2},
$$

where

$$
\Sigma_{p, s, n, b}= \begin{cases}(p-1) \cdot p^{s(n+1)-1} & \text { if } p \mid b \\ -p^{s(n+1)-1} & \text { otherwise. }\end{cases}
$$

Theorem 3.2 For a fixed prime $p \geq 3$ and $b \in \mathbb{Z}_{N_{p}}$, define

$$
n_{p, s, b}:= \begin{cases}\inf \left\{n \in \mathbb{N}:(p-1) p^{s(n+1) / 2-1}>n+1\right\} & \text { if } p \mid b, \\ \inf \left\{n \in \mathbb{N}: p^{s(n+1) / 2-1}>n+1\right\} & \text { otherwise. }\end{cases}
$$

Then for all $n \geq n_{p, s, b}$ we have

$$
M_{p, s, n}(b)\left\{\begin{array}{l}
>0 \text { if } p \mid b \\
<0 \text { otherwise. }
\end{array}\right.
$$


For $p=3$, Li's theorem [4, p. 4, Prop. 1.6] shows that $M_{3,1, n}(b)\left(=M_{3, n}(b)\right)>0$ when $b \equiv 0(\bmod 3)$ for all $n>0$. For $p=3$, when $b \neq 0(\bmod 3)$, we have

Theorem 3.3 Let $b \equiv 1,2(\bmod 3)$ with $b \in \mathbb{Z}_{N_{3}}$. Then

$$
M_{3,1, n}(b)=-\frac{3^{n}}{n+1}(1+o(1)) .
$$

In particular, $M_{3,1, n}(b)<0$ for all $n>0$.

Theorem 3.4 For $p=3, s=2$ and $b \in \mathbb{Z}_{N_{3}}$ we have

$$
M_{3,2, n}(b)= \begin{cases}\frac{2 \cdot 3^{2 n+1}}{n+1}(1+o(1)) & \text { if } 3 \mid b \\ -\frac{3^{2 n+1}}{n+1}(1+o(1)) & \text { otherwise. }\end{cases}
$$

In particular, $M_{3,2, n}(b)>0\left(\right.$ resp. $\left.M_{3,2, n}(b)<0\right)$ when $b \equiv 0(\bmod 3)($ resp. $b \neq \equiv$ $(\bmod 3))$ for all $n>0$.

Theorem 3.5 For $p=5, s=1$ and $b \in \mathbb{Z}_{N_{5}}$ we have

$$
M_{5,1, n}(b)= \begin{cases}\frac{4 \cdot 5^{n}}{n+1}(1+o(1)) & \text { if } 5 \mid b, \\ -\frac{5^{n}}{n+1}(1+o(1)) \text { otherwise. }\end{cases}
$$

In particular, $M_{5,1, n}(b)>0\left(\right.$ resp. $\left.M_{5,1, n}(b)<0\right)$ when $b \equiv 0(\bmod 5)($ resp. $b \not \equiv 0$ (mod 5)) for all $n>0$.

In view of (1.2), we immediately deduce the following from Theorem 3.1:

Corollary 3.5.1 For $p \geq 3$ and $b \in \mathbb{Z}_{N_{p}}$, let $b \equiv j(\bmod p)$. Then we have

$$
\sum_{0 \leq \ell \leq B_{p, s}(b)} t_{b+\ell N_{p}, p, s}=\sum_{0 \leq \ell \leq B_{p, s}(b)}\left[q^{b-j+\ell N_{p}}\right] T_{j, p, s, n}\left(q^{p}\right)=\frac{\Sigma_{p, s, n, b}}{n+1}(1+o(1)),
$$

where $\Sigma_{p, s, n, b}$ is as in Theorem 3.1 and $B_{p, s}(b)=\left\lfloor\left(d_{p, s, n}-b\right) / N_{p}\right\rfloor$.

In particular, noting the fact that the polynomials $T_{j, p, s, n}(q)$ are the polynomials in the first three Borwein conjectures for suitable choices of $j, p$ and $s$ we have, in view of Theorems 3.3-3.5 the following:

Corollary 3.5.2 For $p=3, s=1$ and a fixed $b \in \mathbb{Z}_{N_{3}}$, let $b=3 u+j$ be such that $j \equiv 1,2(\bmod 3)$. Then we have

$0>\sum_{0 \leq \ell \leq B_{3,1}(b)} t_{3 u+1+\ell N_{3}, 3,1}=\sum_{0 \leq \ell \leq B_{3,1}(b)}\left[q^{3 u+\ell N_{3}}\right] B_{n}\left(q^{3}\right)=-\frac{3^{n}}{n+1}(1+o(1))$, 
$0>\sum_{0 \leq \ell \leq B_{3,1}(b)} t_{3 u+2+\ell N_{3}, 3,1}=\sum_{0 \leq \ell \leq B_{3,1}(b)}\left[q^{3 u+\ell N_{3}}\right] C_{n}\left(q^{3}\right)=-\frac{3^{n}}{n+1}(1+o(1))$.

Corollary 3.5.3 For $p=3, s=2$ and a fixed $b \in \mathbb{Z}_{N_{3}}$, let $b=3 u+j$ be such that $j \equiv 0,1,2(\bmod 3)$. Then we have

$$
\begin{aligned}
0<\sum_{0 \leq \ell \leq B_{3,2}(b)} t_{3 u+\ell N_{3}, 3,2} & =\sum_{0 \leq \ell \leq B_{3,2}(b)}\left[q^{3 u+\ell N_{3}}\right] \alpha_{n}\left(q^{3}\right)=\frac{2 \cdot 3^{2 n+1}}{n+1}(1+o(1)), \\
0>\sum_{0 \leq \ell \leq B_{3,2}(b)} t_{3 u+1+\ell N_{3}, 3,2} & =\sum_{0 \leq \ell \leq B_{3,2}(b)}\left[q^{3 u+\ell N_{3}}\right] \beta_{n}\left(q^{3}\right)=-\frac{3^{2 n+1}}{n+1}(1+o(1)), \\
0>\sum_{0 \leq \ell \leq B_{3,2}(b)} t_{3 u+2+\ell N_{3}, 3,2} & =\sum_{0 \leq \ell \leq B_{3,2}(b)}\left[q^{3 u+\ell N_{3}}\right] \gamma_{n}\left(q^{3}\right)=-\frac{3^{2 n+1}}{n+1}(1+o(1)) .
\end{aligned}
$$

Corollary 3.5.4 For $p=5, s=1$ and $b \in \mathbb{Z}_{N_{5}}$, let $b \equiv j(\bmod 5)$. Then we have

$$
\begin{aligned}
\sum_{0 \leq \ell \leq B_{5,1}(b)} t_{b+\ell N, 5,1} & =\sum_{0 \leq \ell \leq B_{5,1}(b)}\left[q^{b-j+\ell N}\right] T_{j, 5,1, n}\left(q^{5}\right) \\
& =\left\{\begin{array}{l}
\frac{4 \cdot 5^{n}}{n+1}(1+o(1))>0 \text { if } 5 \mid b \\
-\frac{5^{n}}{n+1}(1+o(1))<0 \text { otherwise }
\end{array}\right.
\end{aligned}
$$

where $T_{0,5,1, n}(q)=v_{n}(q), T_{1,5,1, n}(q)=\phi_{n}(q), T_{2,5,1, n}(q)=\chi_{n}(q), T_{3,5,1, n}(q)=$ $\psi_{n}(q), T_{4,5,1, n}(q)=\omega_{n}(q)$ are the polynomials in Conjecture 1.3.

Theorem 3.6 Let $p=2,3,5,7,11,13$ and $n$ be sufficiently large. Then we have

$$
\max _{i}\left|t_{i, p, s}\right|=p^{s(n+1)+O(\log n)} .
$$

Theorem 3.7 Let $p>15$ and $n$ be sufficiently large. Then we have

$$
\max _{i}\left|t_{i, p, s}\right| \gtrsim \frac{(1.219 \cdots)^{s(p-1)(n+1)}}{s p^{2} n^{2}}>\frac{p^{s(n+1)-2}}{s n^{2}} .
$$

\section{Li-Wan sieve}

The quantity $M_{p, s, n}\left(k_{1}, k_{2}, \ldots, k_{s} ; b\right)$ is the number of certain type of subsets of $D_{p}^{s}$. As in [4] we apply some elementary character theory to estimate it.

We note that

$$
\rho:=\sum_{\psi \in \hat{G}} \psi
$$


is the regular character of $G$. It is well known that $\rho(g)=0$ for all $g \in G \backslash\{0\}$, and that $\rho(0)=|G|=N_{p}$. Given $0<r \leq\left|D_{p}\right|$, a character $\psi \in \hat{G}$, and $\bar{x}=\left(x_{1}, \ldots, x_{r}\right)$, we set

$$
\prod_{i=1}^{r} \psi\left(x_{i}\right):=f_{\psi}(\bar{x}) \text { and } \mathcal{S}(\bar{x}):=\sum_{i=1}^{r} x_{i} .
$$

Let $Y_{p, s}^{k_{1}, k_{2}, \ldots, k_{s}}$ denote the Cartesian product $\prod_{i=1}^{s} \bar{X}_{p, k_{i}}$. Then we have

$$
\begin{aligned}
& k_{1} ! k_{2} ! \cdots k_{s} ! M_{p, s, n}\left(k_{1}, k_{2}, \ldots, k_{s} ; b\right) \\
& =\frac{1}{N_{p}} \sum_{\left(\bar{x}_{1}, \bar{x}_{2}, \ldots, \bar{x}_{s}\right) \in Y_{p, s}^{k_{1}, k_{2}, \ldots, k_{s}}} \sum_{\psi \in \hat{G}} \psi\left(\mathcal{S}\left(\bar{x}_{1}\right)+\mathcal{S}\left(\bar{x}_{2}\right)+\cdots+\mathcal{S}\left(\bar{x}_{s}\right)-b\right) \\
& =N_{p}^{-1} \prod_{i=1}^{s}\left(\frac{(p-1) N_{p}}{p}\right)_{k_{i}} \\
& \quad+N_{p}^{-1} \sum_{\left(\bar{x}_{1}, \bar{x}_{2}, \ldots, \bar{x}_{s}\right) \in Y_{p, s}^{k_{1}, k_{2}, \ldots, k_{s}}} \sum_{\psi_{0} \neq \psi \in \hat{G}} \psi^{-1}(b) \prod_{i=1}^{s} \psi\left(\mathcal{S}\left(\bar{x}_{i}\right)\right) .
\end{aligned}
$$

In the right-hand side above we interchange the sums to get

$$
\begin{aligned}
& k_{1} ! k_{2} ! \cdots k_{s} ! M_{p, s, n}\left(k_{1}, k_{2}, \ldots, k_{s} ; b\right)=N_{p}^{-1} \prod_{i=1}^{s}\left(\frac{(p-1) N_{p}}{p}\right)_{k_{i}} \\
& \quad+N_{p}^{-1} \sum_{\psi_{0} \neq \psi \in \hat{G}} \psi^{-1}(b) \sum_{\left(\bar{x}_{1}, \bar{x}_{2}, \ldots, \bar{x}_{s}\right) \in Y_{p, s}^{k_{1}, k_{2}, \ldots, k_{s}}} \prod_{i=1}^{s} f_{\psi}\left(\bar{x}_{i}\right) \\
& =N_{p}^{-1} \prod_{i=1}^{s}\left(\frac{(p-1) N_{p}}{p}\right)_{k_{i}} \\
& +N_{p}^{-1} \sum_{\psi_{0} \neq \psi \in \hat{G}} \psi^{-1}(b) \prod_{i=1}^{s}\left(\sum_{\bar{x}_{i} \in \bar{X}_{p, k_{i}}} f_{\psi}\left(\bar{x}_{i}\right)\right) .
\end{aligned}
$$

For a $Y \subset X_{p, k}$ and a character $\psi \in \hat{G}$, set $F_{\psi}(Y):=\sum_{\bar{y} \in Y} f_{\psi}(\bar{y})$. We now have

$$
\begin{aligned}
& k_{1} ! k_{2} ! \cdots k_{s} ! M_{p, s, n}\left(k_{1}, k_{2}, \ldots, k_{s} ; b\right) \\
& =N_{p}^{-1} \prod_{i=1}^{s}\left(\frac{(p-1) N_{p}}{p}\right)_{k_{i}} \\
& \quad+N_{p}^{-1} \sum_{\psi_{0} \neq \psi \in \hat{G}} \psi^{-1}(b) \prod_{i=1}^{s} F_{\psi}\left(\bar{X}_{p, k_{i}}\right) .
\end{aligned}
$$


We now estimate sums of the form $F_{\psi}\left(\bar{X}_{p, k}\right)$. The symmetric group $S_{k}$ acts naturally on $X_{p, k}=D_{p}^{k}$. Let $\tau \in S_{k}$ be a permutation whose cycle decomposition is

$$
\tau=\left(i_{1} i_{2} \cdots i_{a_{1}}\right)\left(j_{1} j_{2} \cdots j_{a_{2}}\right) \cdots\left(\ell_{1} \ell_{2} \cdots \ell_{a_{s}}\right)
$$

where $a_{i} \geq 1,1 \leq i \leq s$. We define

$$
X_{p, k}^{\tau}:=\left\{\left(x_{1}, x_{2}, \ldots, x_{k}\right) \in X_{p, k}: x_{i_{1}}=\cdots=x_{i_{a_{1}}}, \cdots, x_{\ell_{1}}=\cdots=x_{\ell_{a_{s}}}\right\} .
$$

In other words, $X_{p, k}^{\tau}$ is the set of elements in $X_{p, k}$ fixed under the action of $\tau$. Let $C_{k}$ be a set of conjugacy class representatives of $S_{k}$. Let us denote by $C(\tau)$ the number of elements conjugate to $\tau$. Now for any $\tau \in S_{k}$, we have $\tau\left(X_{p, k}\right)=X_{p, k}$. We note that for any pair $\tau, \tau^{\prime}$ of conjugate permutations, and for any $\psi \in \hat{G}$, we have $F_{\psi}\left(\bar{X}_{n, k}^{\tau}\right)=F_{\psi}\left(\bar{X}_{n, k_{i}}^{\tau^{\prime}}\right)$. That is, according to the definitions in [3], $X_{p, k}$ is symmetric and $f_{\psi}$ is normal on $X$. Thus we have the following result which is essentially [3, Proposition 2.8].

Proposition 4.1 We have

$$
F_{\psi}\left(\bar{X}_{p, k}\right)=\sum_{\tau \in C_{k}} \operatorname{sgn}(\tau) C(\tau) F_{\psi}\left(\bar{X}_{p, k}^{\tau}\right) .
$$

\subsection{Some useful lemmas}

The following lemma exhibits the relationship between $F_{\psi}\left(\bar{X}_{p, k}^{\tau}\right)$ and the cycle structure of $\tau$.

Lemma 4.2 Let $\tau \in C_{k}$ be the representative whose cyclic structure is associated with the partition $\left(1^{c_{1}}, 2^{c_{2}}, \ldots k^{c_{k}}\right)$ of $k$. Then we have $F_{\psi}\left(X_{p, k}^{\tau}\right)=$ $\prod_{i=1}^{k}\left(\sum_{a \in D_{p}} \psi^{i}(a)\right)^{c_{i}}$.

Proof Recall that

$$
\begin{aligned}
F_{\psi}\left(X_{p, k}^{\tau}\right) & =\sum_{\bar{x} \in X_{p, k}^{\tau}} \prod_{i=1}^{k} \psi\left(x_{i}\right) \\
& =\sum_{\bar{x} \in X_{p, k}^{\tau}} \prod_{i=1}^{c_{1}} \psi\left(x_{i}\right) \prod_{i=1}^{c_{2}} \psi^{2}\left(x_{c_{1}+2 i}\right) \ldots \prod_{i=1}^{c_{k}} \psi^{k}\left(x_{c_{1}+c_{2} \ldots+k i}\right) \\
& =\prod_{i=1}^{k}\left(\sum_{a \in D_{p}} \psi^{i}(a)\right)^{c_{i}} .
\end{aligned}
$$


Given $\chi \in \hat{G}$ define

$$
s_{D_{p}}(\chi):=\sum_{a \in D_{p}} \chi(a)
$$

Let $N\left(c_{1}, c_{2}, \ldots c_{k}\right)$ denote the number of elements of $S_{k}$ of cycle type $\left(c_{1}, c_{2}, \ldots c_{k}\right)$. It is well known (see, for example, [5]) that

$$
N\left(c_{1}, c_{2}, \ldots c_{k}\right)=\frac{k !}{1^{c_{1}} c_{1} ! 2^{c_{2}} c_{2} ! \cdots k^{c_{k}} c_{k} !} .
$$

Then

Lemma 4.3 We have

$$
F_{\psi}\left(\bar{X}_{p, k}\right)=(-1)^{k} \sum_{\sum_{i} i c_{i}=k} N\left(c_{1}, c_{2}, \ldots, c_{k}\right) \prod_{i=1}^{k}\left(-s_{D_{p}}\left(\psi^{i}\right)\right)^{c_{i}} .
$$

Proof To prove this lemma, we first note that $\operatorname{sgn}(\tau)=(-1)^{k-\sum_{i} c_{i}}$. Also the cyclic structure for every $\tau \in C_{k}$ can be associated to a partition of $k$ of the form $\left(1^{c_{1}}, 2^{c_{2}}, \ldots, k^{c_{k}}\right)$. Hence the right-hand sum in Proposition 4.1 runs over all such partitions of $k$. Noting that the conjugate permutations have same cycle type, and there are exactly $N\left(c_{1}, c_{2}, \ldots, c_{k}\right)$ permutations with cycle type $\left(c_{1}, c_{2}, \ldots c_{k}\right)$, we conclude, in view of Lemma 4.2, that

$$
F_{\psi}\left(\bar{X}_{p, k}\right)=(-1)^{k} \sum_{\sum_{i} i c_{i}=k} N\left(c_{1}, c_{2}, \ldots, c_{k}\right) \prod_{i=1}^{k}\left(-\sum_{a \in D_{p}} \psi^{i}(a)\right)^{c_{i}}
$$

Define the following polynomial in $k$ variables:

$$
Z_{k}\left(t_{1}, \ldots, t_{k}\right):=\sum_{\sum i c_{i}=k} N\left(c_{1}, \ldots, c_{k}\right) t_{1}^{c_{1}} \ldots t_{k}^{c_{k}}
$$

From Lemma 4.3 and (4.5) we immediately see that

Corollary 4.3.1 We have

$$
F_{\psi}\left(\bar{X}_{p, k}\right)=(-1)^{k} Z_{k}\left(-s_{D_{p}}(\psi),-s_{D_{p}}\left(\psi^{2}\right), \ldots,-s_{D_{p}}\left(\psi^{k}\right)\right)
$$

where for $\chi \in \hat{G}, s_{D_{p}}(\chi)$ is as in (4.3).

Thus, it only remains to evaluate the sums $s_{D_{p}}(\chi)$ for $\chi=\psi^{i}, i=1,2, \ldots, k$, and we do this next. Let $o(\chi)$ denotes the order of the character $\chi$. Then 
Lemma 4.4 Let

$$
\delta_{1}^{\psi}(i):= \begin{cases}0 & \text { if } o(\psi) \nmid i \\ -(p-1) N_{p} / p & \text { otherwise }\end{cases}
$$

and

$$
\delta_{2}^{\psi}(i):= \begin{cases}0 & \text { if } o(\psi) \neq 1, p, \\ N_{p} / p & \text { if } o(\psi) / p \mid i \text { and } o(\psi) \nmid i \\ -(p-1) N_{p} / p & \text { if } o(\psi) \mid i\end{cases}
$$

Then

(1) if $p \nmid o(\psi), F_{\psi}\left(\bar{X}_{p, k}\right)=(-1)^{k} Z_{k}\left(\delta_{1}^{\psi}(1), \ldots, \delta_{1}^{\psi}(k)\right)$, and

(2) if $p \mid o(\psi), F_{\psi}\left(\bar{X}_{p, k}\right)=(-1)^{k} Z_{k}\left(\delta_{2}^{\psi}(1), \ldots, \delta_{2}^{\psi}(k)\right)$.

Proof First, observe that $G \backslash D_{p}$ is a subgroup of index $p$. Hence from elementary character theory, we can deduce that

A. if $o(\psi) \neq 1, p$, we have $s_{D_{p}}(\psi)=s_{G}(\psi)-s_{G \backslash D_{p}}(\psi)=0$,

B. if $o(\psi)=1$, we have $s_{D_{p}}(\psi)=\left|D_{p}\right|=(p-1) N_{p} / p$, and

C. if $o(\psi)=p$, we have $s_{D_{p}}(\psi)=-s_{G \backslash D_{p}}(\psi)=-\left|G \backslash D_{p}\right|=-N_{p} / p$.

In order to estimate $F_{\psi}\left(\bar{X}_{p, k}\right)$, we need to consider the following two cases:

Case I: $p \nmid o(\psi)$. In this case, for all $i$ we have $p \nmid o\left(\psi^{i}\right)$ since $o\left(\psi^{i}\right)=$ $o(\psi) /(o(\psi), i)$. Thus from (A) and (B) we see that

$$
s_{D_{p}}\left(\psi^{i}\right)= \begin{cases}(p-1) N_{p} / p & \text { if } o\left(\psi^{i}\right)=1, \\ 0 & \text { otherwise }\end{cases}
$$

which implies (1) in view of Corollary 4.3.1 and the definition of $\delta_{1}^{\psi}(i)$.

Case II: $p \mid o(\psi)$. Here we have the following from (A), (B) and (C):

$$
s_{D_{p}}\left(\psi^{i}\right)= \begin{cases}(p-1) N_{p} / p & \text { if } o\left(\psi^{i}\right)=1 \\ -N_{p} / p & \text { if } o\left(\psi^{i}\right)=p \\ 0 & \text { otherwise }\end{cases}
$$

which implies (2) in view of Corollary 4.3 .1 and the definition of $\delta_{2}^{\psi}(i)$.

\subsection{Some combinatorial functions and estimates}

We now evaluate $Z_{k}\left(\delta_{1}^{\psi}(1), \ldots, \delta_{1}^{\psi}(k)\right)$ and $Z_{k}\left(\delta_{1}^{\psi}(1), \ldots, \delta_{1}^{\psi}(k)\right)$. From (4.4) and (4.5) we immediately deduce the following:

Lemma 4.5 (Exponential generating function) We have

$$
\sum_{k \geq 0} Z_{k}\left(t_{1}, t_{2}, \ldots t_{k}\right) \frac{u^{k}}{k !}=e^{u t_{1}+u^{2} \frac{t_{2}}{2}+\cdots}
$$


The next result follows by substituting special values for the variables $t_{1}, t_{2}, \ldots$ in Lemma 4.5.

Corollary 4.5.1 We have

(1) if $t_{i}=a$ iff $d \mid i$ and $t_{i}=0$ iff $d \nmid i$, then

$$
Z_{k}(\underbrace{0, \ldots, 0}_{d-1}, a, \underbrace{0, \ldots, 0}_{d-1}, a, \ldots)=\left[\frac{u^{k}}{k !}\right] \frac{1}{\left(1-u^{d}\right)^{a / d}}
$$

(2) if $t_{i}=a$ iff $d \mid i$ and $p \cdot d \nmid i$; if $t_{i}=b$ iff $p \cdot d \mid i$; and if $t_{i}=0$ iff $d \nmid i$, then

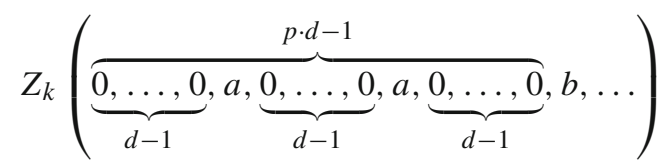

$$
\begin{aligned}
& =\left[\frac{u^{k}}{k !}\right] \frac{1}{\left(1-u^{d}\right)^{a / d}\left(1-u^{p d}\right)^{\frac{b-a}{p d}}} .
\end{aligned}
$$

Proof The proof of this corollary is similar to the case for $p=3$ in $[4$, p. 7, Lemma 2.3].

From Lemma 4.4 and Corollary 4.5.1 we obtain

Lemma 4.6 We have

(1) if $p \nmid o(\psi)$,

$$
F_{\psi}\left(\bar{X}_{p, k}\right)=(-1)^{k}\left[\frac{u^{k}}{k !}\right]\left(1-u^{o(\psi)}\right)^{\frac{(p-1) N_{p}}{p o(\psi)}}
$$

(2) if $p \mid o(\psi)$,

$$
F_{\psi}\left(\bar{X}_{p, k}\right)=(-1)^{k}\left[\frac{u^{k}}{k !}\right] \frac{\left(1-u^{o(\psi)}\right)^{\frac{N_{p}}{o(\psi)}}}{\left(1-u^{o(\psi) / p}\right)^{\frac{N_{p}}{o(\psi)}}}
$$

\section{Proofs of the main results}

Proof of Theorem 3.1 From (4.2) we have

$$
\begin{aligned}
M_{p, s, n}\left(k_{1}, k_{2}, \ldots, k_{s} ; b\right)= & N_{p}^{-1}\left\{\prod_{i=1}^{s}\left(\begin{array}{c}
(p-1) N_{p} / p \\
k_{i}
\end{array}\right)\right. \\
& \left.+P_{k_{1}, k_{2}, \ldots, k_{s}}+Q_{k_{1}, k_{2}, \ldots, k_{s}}+R_{k_{1}, k_{2}, \ldots, k_{s}}\right\},
\end{aligned}
$$


where

$$
\begin{aligned}
P_{k_{1}, k_{2}, \ldots, k_{s}} & =\frac{1}{k_{1} ! k_{2} ! \cdots k_{s} !} \sum_{\psi, p \nmid o(\psi)} \psi^{-1}(b) \prod_{i=1}^{s} F_{\psi}\left(\bar{X}_{p, k_{i}}\right), \\
Q_{k_{1}, k_{2}, \ldots, k_{s}} & =\frac{1}{k_{1} ! k_{2} ! \cdots k_{s} !} \sum_{\psi, o(\psi)=p} \psi^{-1}(b) \prod_{i=1}^{s} F_{\psi}\left(\bar{X}_{p, k_{i}}\right), \\
R_{k_{1}, k_{2}, \ldots, k_{s}} & =\frac{1}{k_{1} ! k_{2} ! \cdots k_{s} !} \sum_{\substack{\psi, p \mid o(\psi) \\
o(\psi) \neq p}} \psi^{-1}(b) \prod_{i=1}^{s} F_{\psi}\left(\bar{X}_{p, k_{i}}\right) .
\end{aligned}
$$

Using Lemma 4.6, we see that

$$
\begin{aligned}
P_{k_{1}, k_{2}, \ldots, k_{s}}= & \frac{(-1)^{k_{1}+k_{2}+\cdots+k_{s}}}{k_{1} ! k_{2} ! \cdots k_{s} !} \sum_{\psi, p \nmid o(\psi)} \psi^{-1}(b) \prod_{i=1}^{s}\left[\frac{u^{k_{i}}}{k_{i} !}\right]\left(1-u^{o(\psi)}\right)^{\frac{(p-1) N_{p}}{p o(\psi)}} \\
Q_{k_{1}, k_{2}, \ldots, k_{s}} & =\frac{(-1)^{k_{1}+k_{2}+\cdots+k_{s}}}{k_{1} ! k_{2} ! \cdots k_{s} !} \sum_{\psi, o(\psi)=p} \psi^{-1}(b) \prod_{i=1}^{s}\left[\frac{u^{k_{i}}}{k_{i} !}\right]\left(\sum_{i=0}^{p-1} u^{i}\right)^{\frac{N_{p}}{p}}, \\
R_{k_{1}, k_{2}, \ldots, k_{s}}= & \frac{(-1)^{k_{1}, k_{2}, \ldots, k_{s}}}{k_{1} ! k_{2} ! \cdots k_{s} !} \sum_{\substack{\psi, p \mid o(\psi) \\
o(\psi) \neq p}} \psi^{-1}(b) \prod_{i=1}^{s}\left[\frac{u^{k_{i}}}{k_{i} !}\right]\left(\sum_{i=0}^{p-1} u^{o(\psi) i / p}\right)^{\frac{N_{p}}{o(\psi)}}
\end{aligned}
$$

Recall that

$$
M_{p, s, n}(b)=\sum_{0 \leq k_{1}, k_{2}, \ldots, k_{s} \leq\left|D_{p}\right|}(-1)^{k_{1}+k_{2}+\cdots+k_{s}} M_{p, s, n}\left(k_{1}, k_{2}, \ldots, k_{s} ; b\right)
$$

Using the well-known fact

$$
\sum_{k=0}^{\left|D_{p}\right|}(-1)^{k}\left(\begin{array}{c}
\left|D_{p}\right| \\
k
\end{array}\right)=0
$$

we see that

$$
\sum_{0 \leq k_{1}, \ldots, k_{s} \leq\left|D_{p}\right|}(-1)^{k_{1}+k_{2}+\cdots+k_{s}} \prod_{i=1}^{s}\left(\begin{array}{c}
(p-1) N_{p} / p \\
k_{i}
\end{array}\right)=\left(\sum_{k=0}^{\left|D_{p}\right|}(-1)^{k}\left(\begin{array}{c}
\left|D_{p}\right| \\
k
\end{array}\right)\right)^{s}=0 .
$$


Thus (5.1), (5.4) and (5.5) yield

$$
\begin{aligned}
M_{p, s, n}(b)= & \frac{1}{N_{p}}\left\{\sum_{0 \leq k_{1}, k_{2}, \ldots, k_{s} \leq\left|D_{p}\right|}(-1)^{k_{1}+k_{2}+\cdots+k_{s}} P_{k_{1}, k_{2}, \ldots, k_{s}}(-1)^{k_{1}+k_{2}+\cdots+k_{s}} Q_{k_{1}, k_{2}, \ldots, k_{s}}\right. \\
& \left.+\sum_{0 \leq k_{1}, k_{2}, \ldots, k_{s} \leq\left|D_{p}\right|}(-1)^{k_{1}+k_{2}+\cdots+k_{s}} R_{k_{1}, k_{2}, \ldots, k_{s}}\right\} . \\
& +\sum_{0 \leq k_{1}, k_{2}, \ldots, k_{s} \leq\left|D_{p}\right|},
\end{aligned}
$$

Given a character $\psi$ of order $p$, there is a unique $x \in\{1, \ldots, p-1\}$ such that for all $y \in \mathbb{Z}_{N_{p}}$, we have $\psi(y)=e^{2 \pi i x y / p}$. Now

Case I: If $p \mid b$. Then

$$
\sum_{\psi, o(\psi)=p} \psi^{-1}(b)=\sum_{x=1}^{p-1} e^{2 \pi i b x / p}=p-1
$$

Case II: If $p \nmid b$. Then, as $x$ runs over elements in $Z_{p}^{\times}$, so does $b x$ and we get

$$
\sum_{\psi, o(\psi)=p} \psi^{-1}(b)=\sum_{x=1}^{p-1} e^{2 \pi i b x / p}=\sum_{x=1}^{p-1} e^{2 \pi i x / p}=-1
$$

So from (5.3) we have

$$
\begin{aligned}
& \sum_{0 \leq k_{1}, k_{2}, \ldots, k_{s} \leq\left|D_{p}\right|}(-1)^{k_{1}+k_{2}+\cdots+k_{s}} Q_{k_{1}, k_{2}, \ldots, k_{s}} \\
= & \left(\sum_{\psi, o(\psi)=p} \psi^{-1}(b)\right)\left(\sum_{0 \leq k_{1}, \ldots, k_{s} \leq\left|D_{p}\right|} \frac{1}{k_{1} ! \cdots k_{s} !} \prod_{i=1}^{s}\left[\frac{u^{k_{i}}}{k_{i} !}\right]\left(\sum_{j=0}^{p-1} u^{j}\right)^{\frac{N_{p}}{p}}\right) \\
= & \left(\sum_{\psi, o(\psi)=p} \psi^{-1}(b)\right) \\
= & \left(\sum_{\psi \leq k_{1}, k_{2}, \ldots, k_{s} \leq\left|D_{p}\right|} \prod_{i=1}^{s}\left[u^{k_{i}}\right]\left(\sum_{j=0}^{p-1} u^{j}\right)^{\frac{N_{p}}{p}}\right) \\
\left.\psi^{-1}(b)\right) & \left(\sum_{k=0}^{\left|D_{p}\right|}\left[u^{k}\right]\left(\sum_{j=0}^{p-1} u^{j}\right)^{\frac{N_{p}}{p}}\right)^{s} \cdot
\end{aligned}
$$


Noting that the sum

$$
\sum_{k=0}^{\left|D_{p}\right|}\left[u^{k}\right]\left(\sum_{j=0}^{p-1} u^{j}\right)^{\frac{N_{p}}{p}}=p^{N_{p} / p}
$$

since it is the sum of all coefficients of the multinomial expansion of $\left(1+u+u^{2}+\right.$ $\left.\cdots+u^{p-1}\right)^{N_{p} / p}$, we obtain the following from (5.7), (5.8) and (5.9):

$$
\begin{aligned}
\Sigma_{p, s, n, b} & :=\sum_{0 \leq k_{1}, k_{2}, \ldots, k_{s} \leq\left|D_{p}\right|}(-1)^{k_{1}+k_{2}+\cdots+k_{s}} Q_{k_{1}, k_{2}, \ldots, k_{s}} \\
& =\left\{\begin{array}{cl}
(p-1) \cdot p^{s N_{p} / p} & \text { if } p \mid b \\
-p^{s N_{p} / p} & \text { otherwise. }
\end{array}\right.
\end{aligned}
$$

Next, we estimate $P_{k_{1}, k_{2}, \ldots, k_{s}}$ and $R_{k_{1}, k_{2}, \ldots, k_{s}}$. Consider

$$
\begin{aligned}
& \left|\sum_{0 \leq k_{1}, k_{2}, \ldots, k_{s} \leq\left|D_{p}\right|}(-1)^{k_{1}+k_{2}+\cdots+k_{s}} R_{k_{1}, k_{2}, \ldots, k_{s}}\right| \\
& =\left|\sum_{\substack{\psi, p \mid o(\psi) \\
o(\psi) \neq p}} \psi^{-1}(b) \sum_{0 \leq k_{1}, k_{2}, \ldots, k_{s} \leq\left|D_{p}\right|} \prod_{i=1}^{s} \frac{1}{k_{i} !}\left[\frac{u^{k_{i}}}{k_{i} !}\right]\left(\sum_{j=0}^{p-1} u^{o(\psi) j / p}\right)^{\frac{N_{p}}{o(\psi)}}\right| \\
& =\left|\sum_{\substack{\psi, p \mid o(\psi) \\
o(\psi)>p}} \psi^{-1}(b) \sum_{0 \leq k_{1}, k_{2}, \ldots, k_{s} \leq\left|D_{p}\right|} \prod_{i=1}^{s}\left[u^{k_{i}}\right]\left(\sum_{j=0}^{p-1} u^{o(\psi) j / p}\right)^{\frac{N_{p}}{o(\psi)}}\right| \\
& \leq \mid \sum_{\substack{p \mid o(\psi) \\
o(\psi)>p}}\left(\sum_{k=0}^{\left|D_{p}\right|}\left[u^{k}\right]\left(\sum_{j=0}^{p-1} u^{o(\psi) j / p}\right)^{N_{p} / o(\psi)}\right)^{s \mid} \\
& \leq \sum_{\substack{p \mid o(\psi) \\
o(\psi)>p}} p^{s N_{p} / o(\psi)}=N_{p} \cdot p^{s N_{p} / 2 p} .
\end{aligned}
$$

Finally, we consider

$$
\begin{aligned}
& \left|\sum_{0 \leq k_{1}, k_{2}, \ldots, k_{s} \leq\left|D_{p}\right|}(-1)^{k_{1}+k_{2}+\cdots+k_{s}} P_{k_{1}, k_{2}, \ldots, k_{s}}\right| \\
& =\left|\sum_{\psi, p \nmid o(\psi)} \psi^{-1}(b) \sum_{0 \leq k_{1}, k_{2}, \ldots, k_{s} \leq\left|D_{p}\right|} \prod_{i=1}^{s} \frac{1}{k_{i} !}\left[\frac{u^{k_{i}}}{k_{i} !}\right]\left(1-u^{o(\psi)}\right)^{\frac{(p-1) N_{p}}{p o(\psi)}}\right|
\end{aligned}
$$




$$
\begin{aligned}
& =\left|\sum_{\substack{\psi, p \nmid o(\psi) \\
o(\psi)>1}} \psi^{-1}(b) \sum_{0 \leq k_{1}, k_{2}, \ldots, k_{s} \leq\left|D_{p}\right|} \prod_{i=1}^{s}\left[u^{k_{i}}\right]\left(1-u^{o(\psi)}\right)^{\frac{(p-1) N_{p}}{p o(\psi)}}\right| \\
& =\left|\sum_{\substack{\psi, p \nmid o(\psi) \\
o(\psi)>1}} \psi^{-1}(b)\left(\sum_{k=0}^{\left|D_{p}\right|}\left[u^{k}\right]\left(1-u^{o(\psi)}\right)^{\frac{(p-1) N_{p}}{p o(\psi)}}\right)^{s}\right| \\
& =0,
\end{aligned}
$$

where the last step is obtained by noting that $\sum_{k=0}^{\left|D_{p}\right|}\left[u^{k}\right]\left(1-u^{j}\right)^{\frac{(p-1) N_{p}}{p j}}$ is the sum of all coefficients of $\left(1-u^{j}\right)^{\frac{(p-1) N_{p}}{p j}}$ which is zero.

Hence (5.6), (5.11), (5.12) and (5.13) yield

$$
\begin{aligned}
\left|M_{p, s, n}(b)-\frac{\Sigma_{p, s, n, b}}{N_{p}}\right| \leq & N_{p}^{-1}\left|\sum_{0 \leq k_{1}, k_{2}, \ldots, k_{s} \leq\left|D_{p}\right|}(-1)^{k_{1}+k_{2}+\cdots+k_{s}} P_{k_{1}, k_{2}, \ldots, k_{s}}\right| \\
& +N_{p}^{-1}\left|\sum_{0 \leq k_{1}, k_{2}, \ldots, k_{s} \leq\left|D_{p}\right|}(-1)^{k_{1}+k_{2}+\cdots+k_{s}} R_{k_{1}, k_{2}, \ldots, k_{s}}\right| \\
\leq & p^{s N_{p} / 2 p},
\end{aligned}
$$

which yields the theorem.

Proof of Theorem 3.2 To prove this theorem, we need to consider two cases.

Case I: $p \mid b$. In this case, from Theorem 3.1 we have

$$
\Sigma_{p, s, n, b}=(p-1) \cdot p^{s(n+1)} .
$$

Thus the main term in Theorem 3.1 dominates the error provided

$$
\frac{(p-1) \cdot p^{s(n+1)-1}}{n+1}>p^{s(n+1) / 2}
$$

It is now clear that for all $n \geq \inf \left\{n \in \mathbb{N}:(p-1) p^{s(n+1) / 2-1}>n+1\right\}, M_{p, s, b}(b)>0$ when $p \mid b$.

Case II: $p \nmid b$. In this case, from Theorem 3.1 we have

$$
\Sigma_{p, s, n, b}=-p^{s(n+1)} .
$$

Thus the absolute value of the main term in Theorem 3.1 dominates the error provided

$$
\frac{p^{s(n+1)-1}}{n+1}>p^{s(n+1) / 2} .
$$


It is now clear that for all $n \geq \inf \left\{n \in \mathbb{N}: p^{s(n+1) / 2-1}>n+1\right\}, M_{p, s, n}(b)<0$ when $p \nmid b$. This proves the theorem.

Proof of Theorem 3.3 The first part of the theorem follows from Theorem 3.1 by choosing $p=3$ and $s=1$. For the other part, we use Theorem 3.2. Thus the smallest $n_{3,1, b} \in \mathbb{N}$ for which

$$
3^{(n+1) / 2-1}>n+1
$$

holds true is $n_{3,1, b}=4$. Thus for all $n \geq 4$ we have $M_{3,1, n}(b)<0$. Also by direct computation, one shows that $M_{3,1, n}(b)<0$ for all $n<4$. Indeed, using Wang's result [6], one immediately concludes that $M_{3,1, n}(b)<0$ without any of the above analysis.

Proof of Theorem 3.4 The first part of this theorem follows directly from Theorem 3.1 by choosing $p=3$ and $s=2$.

For the other part, we use Theorem 3.2. Thus in the case $b \equiv 0(\bmod 3)$ we have

$$
2 \cdot 3^{n}>n+1
$$

for all $n \in \mathbb{N}$. Hence $M_{3,2, n}>0$ for all $n \in \mathbb{N}$. In the case $b \not \equiv 0(\bmod 3)$ we have

$$
3^{n}>n+1
$$

holds true for all $n \in \mathbb{N}$. Hence $M_{3,2, n}<0$ for all $n \in \mathbb{N}$.

Proof of Theorem 3.5 The first part of this theorem follows directly from Theorem 3.1 by choosing $p=5$ and $s=1$.

For the other part, we use Theorem 3.2. Thus in the case $b \equiv 0(\bmod 5)$ we have

$$
4 \cdot 5^{(n+1) / 2-1}>n+1
$$

for all $n \in \mathbb{N}$. Hence $M_{5,1, n}>0$ for all $n \in \mathbb{N}$. In the case $b \not \equiv 0(\bmod 5)$ we have

$$
5^{(n+1) / 2-1}>n+1
$$

holds true for all $n \geq 3$. By direct computation one checks that $M_{5,1, n}<0$ for all $n<3$. Hence $M_{5,1, n}<0$ for all $n \in \mathbb{N}$.

Proof of Theorem 3.6 Using Cauchy's formula we see that

$$
\begin{aligned}
\left|t_{j, p, s}\right| & =\left|\frac{1}{2 i \pi} \int_{|q|=1} \frac{T_{p, s, n}(q)}{q^{j+1}} d q\right| \\
& \leq \frac{1}{2 \pi} \max _{|q|=1}\left|T_{p, s, n}(q)\right| \int_{|q|=1} \frac{|d q|}{|q|^{j+1}}=\max _{|q|=1}\left|T_{p, s, n}(q)\right| .
\end{aligned}
$$


On the other hand we have

$$
\max _{|q|=1}\left|T_{p, s, n}(q)\right| \leq \sum_{0 \leq j \leq N_{s, n}}\left|t_{j, p, s}\right| \leq\left(d_{p, s, n}+1\right) \max _{j}\left|t_{j, p, s}\right|
$$

Since $d_{p, s, n} \leq s p^{2} n^{2},(5.16)$ and (5.17) imply

$$
\log _{p} \max _{j}\left|t_{j, s, n}\right|=\log _{p} \max _{|q|=1}\left|T_{p, s, n}(q)\right|+O(\log n) .
$$

Thus the theorem follows if we show that

$$
\log _{p} \max _{|q|=1}\left|T_{p, s, n}(q)\right|=s(n+1)+O(\log n) .
$$

We note that

$$
\max _{|q|=1}\left|T_{p, s, n}(q)\right|=\max _{|q|=1}\left|\prod_{j=1}^{n}\left(1-q^{j}\right)\right|^{s}=\left(\max _{|q|=1}\left|\prod_{j=0}^{n} \prod_{k=1}^{p-1}\left(1-q^{p j+k}\right)\right|\right)^{s} .
$$

From [2, Theorem 1, p. 229], we have

$$
\log _{p} \max _{|q|=1}\left|\prod_{j=0}^{n} \prod_{k=1}^{p-1}\left(1-q^{p j+k}\right)\right|=n+1+O\left(\frac{1}{n}\right)
$$

Now the estimate (5.19) and thus the theorem follow from (5.18), (5.20) and (5.21).

Proof of Theorem 3.7 We have

$$
\max _{|q|=1}\left|T_{p, s, n}(q)\right| \leq \sum_{0 \leq j \leq N_{s, n}}\left|t_{j, p, s}\right| \leq\left(d_{p, s, n}+1\right) \max _{j}\left|t_{j, p, s}\right|
$$

which implies since $d_{p, s, n} \leq s p^{2} n^{2}$ that

$$
\max _{j}\left|t_{j, p, s}\right| \geq \frac{1}{d_{p, s, n}+1} \max _{|q|=1}\left|T_{p, s, n}(q)\right|>\frac{1}{s p^{2} n^{2}} \max _{|q|=1}\left|T_{p, s, n}(q)\right| .
$$

From [2, Theorem 2, p. 229] we have

$$
\max _{|q|=1}\left|\prod_{j=0}^{n} \prod_{k=1}^{p-1}\left(1-q^{p j+k}\right)\right| \gtrsim(1.219 \cdots)^{(p-1)(n+1)}>p^{n+1}
$$


Since

$$
\max _{|q|=1}\left|T_{p, s, n}(q)\right|=\left(\max _{|q|=1}\left|\prod_{j=0}^{n} \prod_{k=1}^{p-1}\left(1-q^{p j+k}\right)\right|\right)^{s}
$$

using (5.24) in (5.23) yields

$$
\max _{j}\left|t_{j, p, s}\right| \gtrsim \frac{(1.219 \cdots)^{s(p-1)(n+1)}}{s p^{2} n^{2}}>\frac{p^{s(n+1)-2}}{s n^{2}} .
$$

Acknowledgements The authors thank George Andrews, Peter Paule, Qing Xiang and Cai-Heng Li for their feedback. We also thank the anonymous referee for valuable suggestions and feedback.

Funding Open access funding provided by Austrian Science Fund (FWF).

Open Access This article is licensed under a Creative Commons Attribution 4.0 International License, which permits use, sharing, adaptation, distribution and reproduction in any medium or format, as long as you give appropriate credit to the original author(s) and the source, provide a link to the Creative Commons licence, and indicate if changes were made. The images or other third party material in this article are included in the article's Creative Commons licence, unless indicated otherwise in a credit line to the material. If material is not included in the article's Creative Commons licence and your intended use is not permitted by statutory regulation or exceeds the permitted use, you will need to obtain permission directly from the copyright holder. To view a copy of this licence, visit http://creativecommons.org/licenses/by/4.0/.

\section{References}

1. Andrews, G.: On a conjecture of Peter Borwein. J. Symbol. Comput. 20, 487-501 (1995)

2. Borwein, P.: Some restricted partition functions. J. Number Theory 45, 228-240 (1993)

3. Li, J., Wan, D.: A new sieve for distinct coordinate counting. Sci. China Math. (Springer) 53(9), 23512362 (2010)

4. Li, J.: On the Borwein conjecture. Int. J. Number Theory 16(5), 1053-1066 (2020)

5. Stanley, R.P.: Enumerative Combinatorics, vol. 1. Cambridge University Press, Cambridge (1997)

6. Wang, C.: An analytic proof of the Borwein conjecture. arXiv: 1901.10886 (2019)

7. Zaharescu, A.: Borwein's conjecture on average over arithmetic progression. Ramanujan J. 11, 95-102 (2006)

Publisher's Note Springer Nature remains neutral with regard to jurisdictional claims in published maps and institutional affiliations. 